Article

\title{
Sensor Applications of Soft Magnetic Materials Based on Magneto-Impedance, Magneto-Elastic Resonance and Magneto-Electricity
}

\author{
Alfredo García-Arribas ${ }^{1,2, *}$, Jon Gutiérrez ${ }^{1,2}$, Galina V. Kurlyandskaya ${ }^{1}$, \\ José M. Barandiarán ${ }^{1,2}$, Andrey Svalov ${ }^{1}$, Eduardo Fernández ${ }^{1}$, Andoni Lasheras ${ }^{1}$, \\ David de $\operatorname{Cos}^{3}$ and Iñaki Bravo-Imaz ${ }^{4}$ \\ 1 Department of Electricity and Electronics, Basque Country University (UPV/EHU), \\ P.O. Box 644, 48080 Bilbao, Spain; E-Mails: jon@we.lc.ehu.es (J.G.); \\ galina@we.lc.ehu.es (G.V.K.); manub@we.lc.ehu.es (J.M.B.); andrey.svalov@ehu.es (A.S.); \\ eduardo.fernandez@ehu.es (E.F.); andoni.lasheras@ehu.es (A.L.) \\ 2 BCMaterials, Technological Park of Biscay, No 500, 48160 Derio, Spain \\ 3 ESS Bilbao, Paseo de Landabarri, 48940 Leioa, Spain; E-Mail: dce@we.lc.ehu.es \\ 4 Intelligent Information Systems, IK4-Tekniker, 20600 Eibar, Spain; \\ E-Mail: inaki.bravo@tekniker.es \\ * Author to whom correspondence should be addressed; E-Mail: alf@we.lc.ehu.es; \\ Tel.: +34-946-015-307; Fax: +34-946-013-071.
}

Received: 21 January 2014; in revised form: 2 April 2014 / Accepted: 15 April 2014 / Published: 25 April 2014

\begin{abstract}
The outstanding properties of selected soft magnetic materials make them successful candidates for building high performance sensors. In this paper we present our recent work regarding different sensing technologies based on the coupling of the magnetic properties of soft magnetic materials with their electric or elastic properties. In first place we report the influence on the magneto-impedance response of the thickness of Permalloy films in multilayer-sandwiched structures. An impedance change of $270 \%$ was found in the best conditions upon the application of magnetic field, with a low field sensitivity of $140 \% /$ Oe. Second, the magneto-elastic resonance of amorphous ribbons is used to demonstrate the possibility of sensitively measuring the viscosity of fluids, aimed to develop an on-line and real-time sensor capable of assessing the state of degradation of lubricant oils in machinery. A novel analysis method is shown to sensitively reveal the changes of the damping parameter of the magnetoelastic oscillations at the resonance as a function of the oil viscosity. Finally, the properties and performance of magneto-electric
\end{abstract}


laminated composites of amorphous magnetic ribbons and piezoelectric polymer films are investigated, demonstrating magnetic field detection capabilities below $2.7 \mathrm{nT}$.

Keywords: magneto-impedance; magneto-elasticity; magneto-electricity; magnetic sensors

\section{Introduction}

Magnetic sensors were introduced long ago to the field of electrical engineering and biomedical research. There are many types of magnetic field sensors. Many topical reviews have summarized the most important parameters and properties of magnetic field sensors, like bandwidth, full scale range, linearity, hysteresis, temperature coefficient of sensitivity, bias stability, offset features, long term stability, noise, resistance to the environment factors, power consumption, size, cost, etc. A detailed classification of the magnetic sensors based both on the employed materials and effects is given in [1-3]. For magnetic sensors based on semiconductors, superconducting quantum interferometers (SQUIDs) and recording heads see also [4,5]. Interesting examples of more specific descriptions of selected types of magnetic sensors based on the Hall effect, fluxgate sensing, magnetoresistance, giant magnetoimpedance (MI), and spin-polarized can be found in [2,6-8].

The coupling between the magnetic properties of soft magnetic materials and their elastic or electrical response gives rise to different phenomena that can be used for sensing purposes. On the one hand, the magneto-impedance (MI) accounts for the skin-effect-induced change of impedance caused by an applied magnetic field. It allows developing sensible magnetic field sensors for applications such as geomagnetism and biosensing, among many others. On the other hand, magnetoelastic resonance (MR) takes place when a mechanical resonant response is excited through the magnetostrictive effect of soft magnetic materials. The effect can be used to sense any magnitude that influences the resonant behavior and, as the resonance can be excited remotely, this effect is very convenient for non-contact applications. Finally, by combining those magnetostrictive amorphous ferromagnetic ribbons with piezoelectric materials, we have fabricated magneto-electric (ME) laminated composites, that show an extremely high sensitivity for magnetic field detection.

In close collaboration with the BCMaterials Research Center, and other Technological Centers of the Basque Country, the Group of Magnetism and Magnetic Materials of the Universidad del País Vasco (UPV/EHU) maintains an intense activity devoted to the development of sensors based on the above-mentioned magnetic materials and effects. The more recent results are described, for each of the research lines, in the following sections.

\section{Magneto-Impedance (MI) Sensors}

The magneto-impedance (MI) is the change of the complex impedance $(Z)$ that ferromagnetic conductors experience when an external magnetic field $(H)$ is applied and a high frequency $(\omega)$ alternating current flows through them. Great efforts were made in the last years in the development of magnetic thin-film-based sensitive elements with controllable properties for MI applications, including low magnetic field detection and biosensing [9-11]. Some excellent reviews are available [12-14]. 
One of the most studied MI thin film materials is the $\mathrm{Fe}_{19} \mathrm{Ni}_{81}$ Permalloy (Py), a soft magnetic material with low coercivity, high magnetic permeability and saturation magnetization [15]. Many sensor applications (for example, flat inductors, magnetoelectronic devices and MI itself) require FeNi thin film elements with thickness of the order of microns to obtain the desired properties [16-18]. In particular, MI is enhanced in sandwiched structures with a central non-magnetic conductor [19]. The magnetic softness of thin Py films depends on their thickness due to the crucial role of a transition into a "transcritical" state [18,20]. It manifests by the appearance of a perpendicular magnetic anisotropy component, high coercivity, and striped magnetic domain structure [21]. Different research groups have proposed the nanostructuring of Py films in order to solve this problem $[11,18]$. The effectiveness of using magnetic $[\mathrm{Py} / \mathrm{X}]_{n}$ multilayers $(\mathrm{X}-$ is a separating layer made of $\mathrm{Cu}, \mathrm{Ti}, \mathrm{Ag}$ or Gd and $n$ the number of layers in the multilayered structure) was carefully analyzed [11,18,22,23]. This strategy was also successfully applied for $\mathrm{Fe}_{72.5} \mathrm{Cu}_{1.1} \mathrm{Nb}_{1.9} \mathrm{Mo}_{1.5} \mathrm{Si}_{4.2} \mathrm{~B}_{8.7}$ alloys [24]. In contrast, the thickness of the magnetic layers in the $[\mathrm{Py} / \mathrm{X}]_{n}$ multilayers has not been systematically analysed yet, although some interesting structural features observed in Py nanostructures can be used for the improvement of their magnetic properties [25].

In this section, we describe the preparation and characterization of MI samples containing Py/Ti multilayer structures with different Py layer thicknesses. The samples have a sandwiched structure, using a layer of $\mathrm{Cu}$ as central conductor between two Py/Ti multilayer structures. Although the Py layers have different thickness, the same total thickness of the top and bottom structures of the MI sandwich is maintained. We aim to obtain very high MI sensitivity with respect to the applied magnetic field systematically, with the purpose of designing optimized nanostructured multilayers sensitive elements to detect small magnetic fields.

\subsection{Magneto-Impedance Sample Preparation}

The multilayer sandwiched (MS) structures, where the magnetic layers are composed of Py/Ti multilayers, and the central non-magnetic layer is made of copper, were deposited by Direct Current (DC) magnetron sputtering onto glass substrates. The role of the background and working Ar pressure was previously studied to obtain the optimum deposition conditions [21]. In the present studies, a background pressure of $3 \times 10^{-7}$ mbar and working Ar pressure of $3.8 \times 10^{-3}$ mbar were used. The $[\mathrm{Py} / \mathrm{Ti}]_{n}$ magnetic layers at the top and bottom of the sandwiched structures were composed of equal thickness $\mathrm{Fe}_{80} \mathrm{Ni}_{20}$ layers, separated by $6 \mathrm{~nm}$ Ti layers. The thickness of the Ti spacer was optimized in a previous study of $\mathrm{FeNi} / \mathrm{Ti} / \mathrm{FeNi}$ bilayers [23]. In all cases, the sum of the thickness for all the Py layers in the structure was equal to $300 \mathrm{~nm}$. The number $(n)$ of Py and Ti layers in the multilayer was adjusted accordingly.

A transverse magnetic anisotropy was induced during the deposition process by the application of an in-plane constant magnetic field of 250 Oe. The samples were shaped during deposition as $10 \mathrm{~mm}$ long and $0.5 \mathrm{~mm}$ wide rectangles using metallic masks. The long sides of the samples were oriented perpendicularly to the direction of the applied magnetic field. Therefore, the induced magnetic anisotropy axis was formed parallel to the short side of the stripe in all samples. Figure 1 and Table 1 give a description of the studied MS structures. 
Figure 1. Schematic description of the Magneto-Impedance multilayer sandwiched (MS) structure used in this work. Each sample has different Py thickness $(\Delta)$ and different number of repetitions of the [Py/Ti] structure (3, 6 and 12 for samples MSPy100, MSPy50, and MSPy25, respectively), being the $\mathrm{Cu}$ central conductor of $500 \mathrm{~nm}$ in all cases.

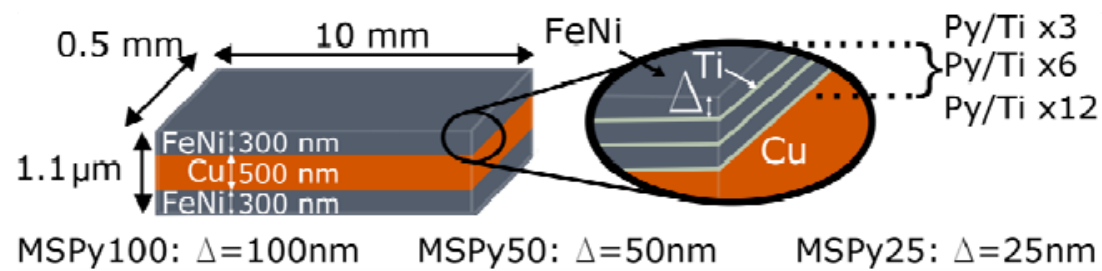

Table 1. Description of the samples and summary of selected properties.

\begin{tabular}{lccc}
\hline Sample structure & {$[\mathrm{Py} / \mathrm{Ti}]_{3} / \mathrm{Cu} /[\mathrm{Ti} / \mathrm{Py}]_{3}$} & {$[\mathrm{Py} / \mathrm{Ti}]_{6} / \mathrm{Cu} /[\mathrm{Ti} / \mathrm{Py}]_{6}$} & {$[\mathrm{Py} / \mathrm{Ti}]_{12} / \mathrm{Cu} /[\mathrm{Ti} / \mathrm{Py}]_{12}$} \\
Sample identifier & $\mathrm{MSPy} 100$ & $\mathrm{MSPy} 50$ & $\mathrm{MSPy} 25$ \\
FeNi layer thickness & $100 \mathrm{~nm}$ & $50 \mathrm{~nm}$ & $25 \mathrm{~nm}$ \\
$R_{\mathrm{DC}}(\Omega)$ & 1.65 & 2.51 & 2.25 \\
$H_{c}(\mathrm{Oe})$ & 0.15 & 0.20 & 0.40 \\
$H_{k}(\mathrm{Oe})$ & 2.8 & 4.0 & 4.5 \\
$(\Delta Z / Z)_{\max }(\%)$ & 270 & 210 & 170 \\
$(\Delta R / R)_{\max }(\%)$ & 580 & 420 & 380 \\
$s[(\Delta Z / Z) / \Delta H]_{\max }(\% / \mathrm{Oe})$ & 140 & 110 & 60 \\
$s[(\Delta R / R) / \Delta H]_{\max }(\% / \mathrm{Oe})$ & 230 & 160 & 100 \\
\hline
\end{tabular}

$R_{\mathrm{DC}}-\mathrm{DC}$ resistance of the sample; $H_{c}$-coercive field; $H_{k}$-anisotropy field; $(\Delta Z / Z)_{\max } ;(\Delta R / R)_{\max }-$ maximum values of the MI ratio for the absolute value of the impedance and for the real part respectively. $s[(\Delta Z / Z) / \Delta H]_{\max }, s[(\Delta R / R) / \Delta H]_{\max }$-maximum sensitivities of both ratios.

The in-plane hysteresis loops were measured using a vibrating sample magnetometer (VSM). The importance of the dispersion of the easy magnetization axes was widely discussed in the literature for the case of MI in amorphous ribbons [26]. In this work, fine features of the transverse magnetic anisotropy induced in Py/Ti multilayers were estimated following the procedure described in [27], i.e., by measuring the sample's second harmonic magnetic response to the application of a small amplitude Alternating Current (AC) exciting field, with decreasing and increasing fields denoted as "Down" and "Up". Even in the case of wide anisotropy distributions, the measured experimental distribution parameters give useful information about the magnetization processes in the multilayered structures.

The MI measurements were performed using Radio Frequency (RF) techniques, which are described in detail elsewhere [28]. The samples were glued into a $50 \Omega$ microstrip line using silver paint. The total impedance $(Z)$ and the real $(R)$ component were obtained from the measured $S_{11}$ parameters after calibration and mathematical subtraction of the test fixture contributions. The test fixture was placed inside the central region of a pair of Helmholtz coils and the full cycle of the impedance change was measured for the interval \pm 150 Oe of the external applied magnetic field. An exciting current in the frequency range of $300 \mathrm{kHz}$ to $300 \mathrm{MHz}$ was used. The frequency of $300 \mathrm{MHz}$ is low enough to assure that resonance effects were not present and that the quasi-static processes dominated the MI behavior [29]. The MI ratios $\Delta Z / Z_{\text {sat }}$ and $\Delta R / R_{\text {sat }}$ were defined with respect to the magnetic field $H_{\text {sat }}=150 \mathrm{Oe}$, at which the samples were magnetically saturated. MI was 
defined as follows: $\Delta Z / Z_{\text {sat }}=100 \times\left[Z(H)-Z\left(H_{\text {sat }}\right)\right] / Z\left(H_{\text {sat }}\right)$ for the total impedance and $\Delta R / R=100 \times\left[R(H)-R\left(H_{\text {sat }}\right)\right] / R\left(H_{\text {sat }}\right)$ for the real component of the impedance. The MI sensitivities were defined as $s\left[\left(\Delta Z / Z_{s a t}\right) / \Delta H\right]$ and $s\left[\left(\Delta R / R_{\text {sat }}\right) / \Delta H\right]$, with $\Delta H=0.1$ Oe. The direct current (DC) resistance $R_{\mathrm{DC}}$ was measured by the four contacts technique.

\subsection{Results and Discussion}

Figure 2 shows the hysteresis loops of the MS structures obtained with a VSM. The magnetic field was applied in plane and along the short side of the rectangular sample, i.e., along the easy magnetization axis. The shape of the hysteresis loops confirms the formation of an in-plane, transverse induced magnetic anisotropy. All the MS structures are very soft ferromagnets with well-defined magnetic anisotropy, close to uniaxial. Sample MSPy50 (Py layers $50 \mathrm{~nm}$ thick) has the smallest coercive field of 0.15 Oe. Samples MSPy100 and MSPy25, composed of 25 and $100 \mathrm{~nm}$ thick Py layers, have respectively coercive fields of 0.4 Oe and 0.2 Oe. The anisotropy fields $\left(H_{k}\right)$, listed in Table 1, have been obtained from the anisotropy distribution measurements corresponding to the maxima of the anisotropy distribution shown in Figure 3. These values are in close agreement with the anisotropy fields estimated from the $M(H)$ hysteresis loops measured by VSM (Figure 2). Sample MSPy50 has a narrower anisotropy distribution than sample MSPy100, with larger intensity peaks, meaning that the sample has a better defined transverse anisotropy.

Figure 2. Vibrating sample magnetometer (VSM) hysteresis loops measurements, with the applied magnetic field perpendicular to the easy axis.

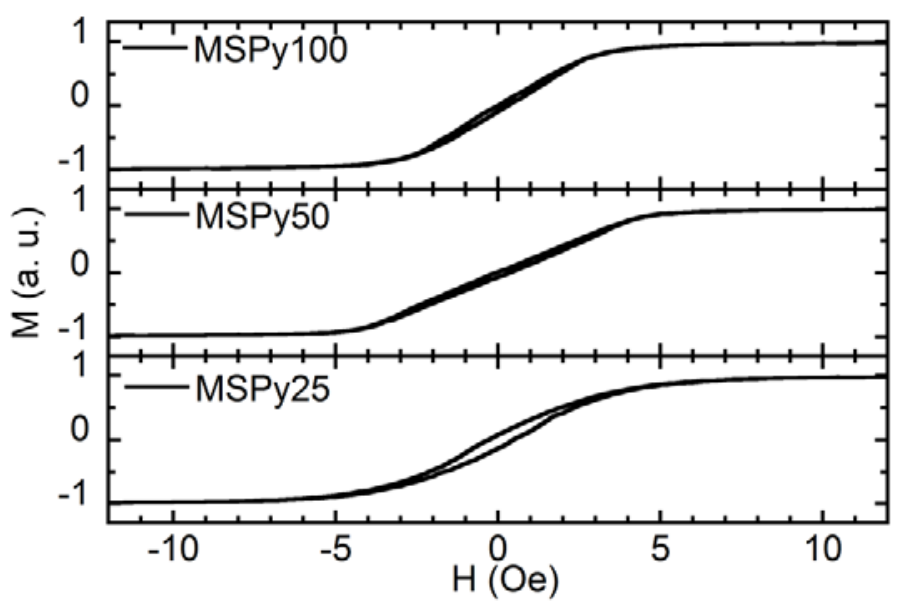

Several factors influence the magnetic behavior of the samples and must be considered. On the one hand, the magnetic properties of each individual layer must be taken into account. The type of domain walls depends on the thickness of the Py films as studied in [25,30]. A single layered $100 \mathrm{~nm}$ thick Py film displays two types of domain walls: 180 Néel walls with cross ties and stray-field free asymmetric mobile-vortex Bloch walls. In the $50 \mathrm{~nm}$ and $25 \mathrm{~nm}$ thick films the magnetic domains with in-plane orientation of the magnetization are separated by Néel walls with cross-ties. The different type of domain wall may surely influence the interaction between layers, as discussed in [31] for the case of bilayers. The advantages of weak interlayer interactions for maintaining a good magnetic softness are evident for certain thicknesses of the non-magnetic spacer. 
Figure 3. Distribution of perpendicular anisotropies from the second harmonic response.

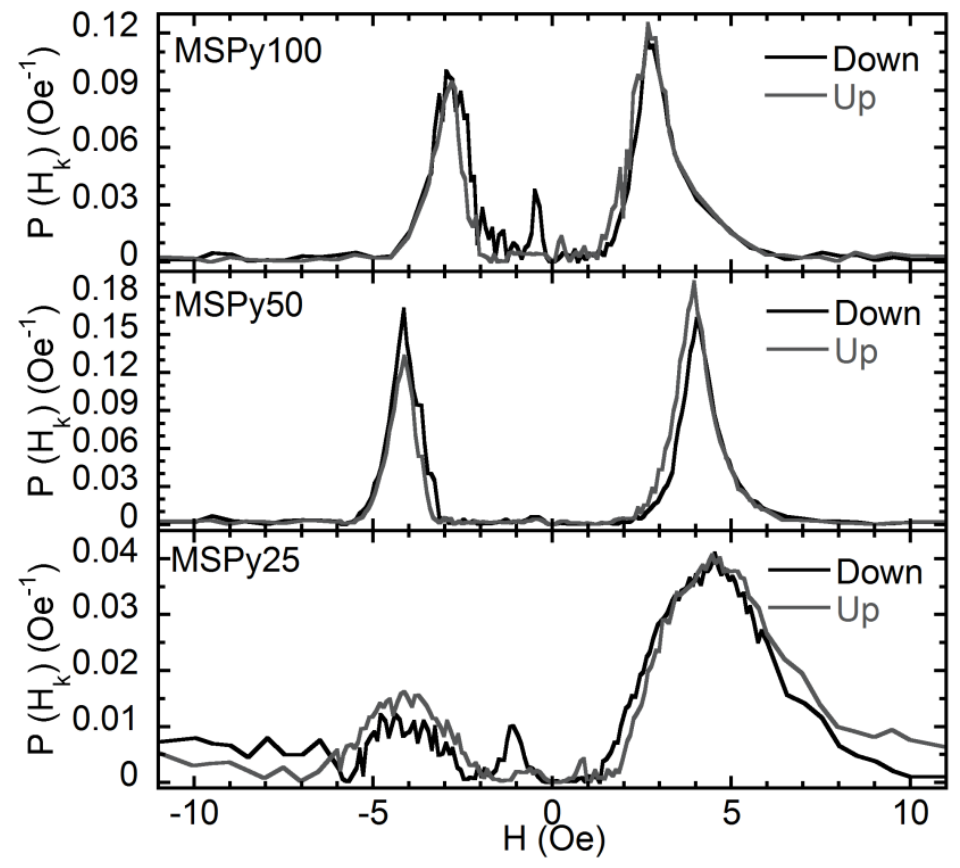

On the other hand, the different contributions of the shape anisotropy to the effective anisotropy of the narrow $[\mathrm{Py} / \mathrm{Ti}]_{n} / \mathrm{Cu} /[\mathrm{Py} / \mathrm{Ti}]_{n}(n=3,6,12)$ stripes can play a decisive role. The easy magnetization axis (EMA) induced during preparation is oriented along the short side of the stripe. The induced anisotropy is therefore competing with the shape anisotropy for which the minimum free energy corresponds to the position along the sample's length [32]. The results of the magnetic measurements confirmed that the mean anisotropy field is larger for the multilayers with thinner Py layers (and larger number of layers).

Moreover, as schematized in Figure 4, each magnetic layer except the first and the last, have two nearest neighbors to close the magnetic flux and decrease the density of the magnetostatic energy at the surfaces perpendicular to the EMA. As the number of layers increases, this circumstance is more favorable, increasing the magnetic field that is necessary in order to rotate the magnetization towards the hard magnetization axis. At the same time, a large number of interfaces is always a source of magnetic inhomogeneities as can be seen from the analysis of the anisotropy distribution of sample MSPy25 in Figure 3.

Figure 5 displays the MI ratios and sensitivities obtained for the MS structures as a function of the external magnetic field at $40 \mathrm{MHz}$. The two-peaked shape of the MI curves is in accordance with the magnetic measurements. Samples MSPy50 and MSPy25 have similar anisotropy fields, so their MI ratios should be similar if we take into account only the MI dependence on the transverse magnetic permeability. Besides, sample MSPy25 has a smaller $R_{\mathrm{DC}}=2.25 \Omega$ compared to the $2.51 \Omega$ of sample MSPy50. The MI ratio would be higher for sample MSPy25 with both samples having the same $\Delta Z$ value. Despite all the advantages that sample MSPy25 should have, at $40 \mathrm{MHz}$, sample MSPy50 shows a higher MI ratio compared with sample MSPy25. The MI sensitivity measured for the sample MSPy50 is also larger than in sample MSPy25. This is related to the higher peak widths of the anisotropy distribution of MSPy25 sample (Figure 3) that makes its MI peak less sharp and therefore decreases the sensitivity. Sample MSPy100 has the best MI response due to the combination of low 
anisotropy field and rather narrow anisotropy distribution. A lower anisotropy field implies a larger transverse magnetic permeability that increases the MI ratio and MI sensitivity. The maximum MI ratio in sample MSPy100 is indeed larger than in sample MSPy50.

Figure 4. Schematic view of the closing magnetic flux at the lateral edges of the $[\mathrm{Py}(100 \mathrm{~nm}) / \mathrm{Ti}]_{3}$ and $[\mathrm{Py}(50 \mathrm{~nm}) / \mathrm{Ti}]_{6}$ multilayers.
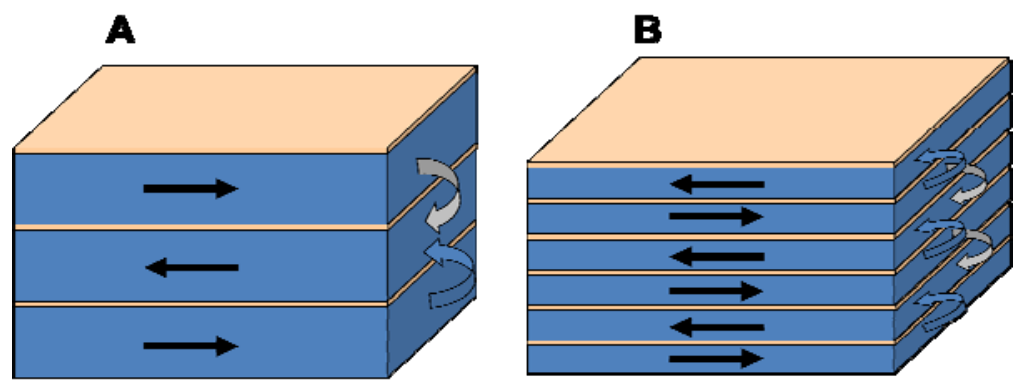

Figure 5. Relative magneto-impedance and sensitivity as a function of the applied magnetic field at $40 \mathrm{MHz}$.

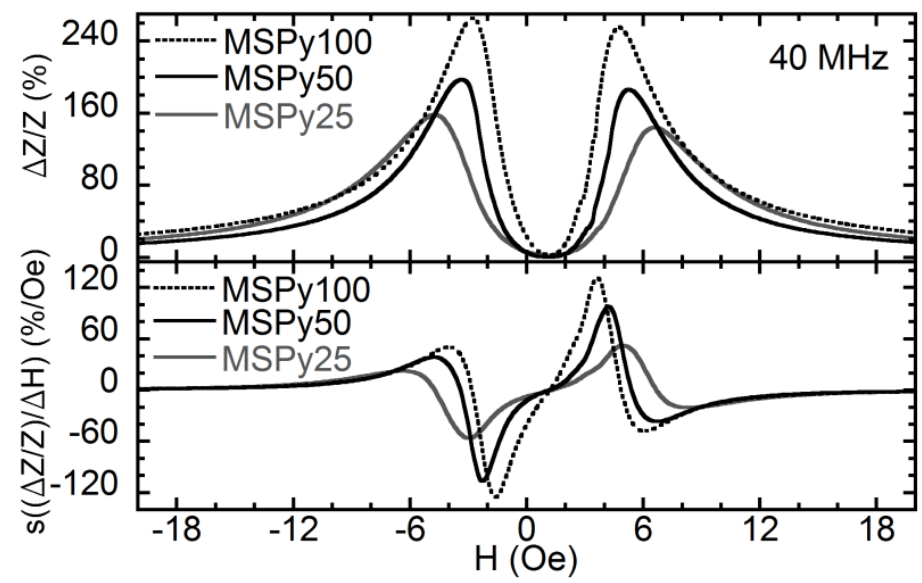

The change in impedance $\Delta Z$ at $40 \mathrm{MHz}$ is slightly larger in sample MSPy50 than in sample MSPy100. However, sample MSPy100 has a smaller DC resistance of $1.65 \Omega$ which makes its impedance variation larger. The MI sensitivity is larger in sample MSPy100 than in MSPy50, as it can be expected since sample MSPy100 has a larger MI ratio and lower anisotropy field. The behavior of the maximum values of the MI ratio and the MI sensitivities in the whole range measured frequencies are shown in Figure 6. All the samples show quite similar behavior. The MI ratios at frequencies over $200 \mathrm{MHz}$ are almost the same for all cases under consideration. At frequencies below $200 \mathrm{MHz}$, sample MSPy100 has the best MI response and MI sensitivity. The maximum sensitivity of 225\%/Oe at $160 \mathrm{MHz}$ was observed for the real part of the impedance. 
Figure 6. Frequency dependence of the maximum value of the $\Delta Z / Z$ ratio, maximum value of the $\Delta R / R$ ratio, maximum value of the sensitivity $s[(\Delta Z / Z) / \Delta H]$ and maximum value of the sensitivity of the real part of the impedance $s[(\Delta R / R) / \Delta H]$ for the three multilayer sandwiched structures.

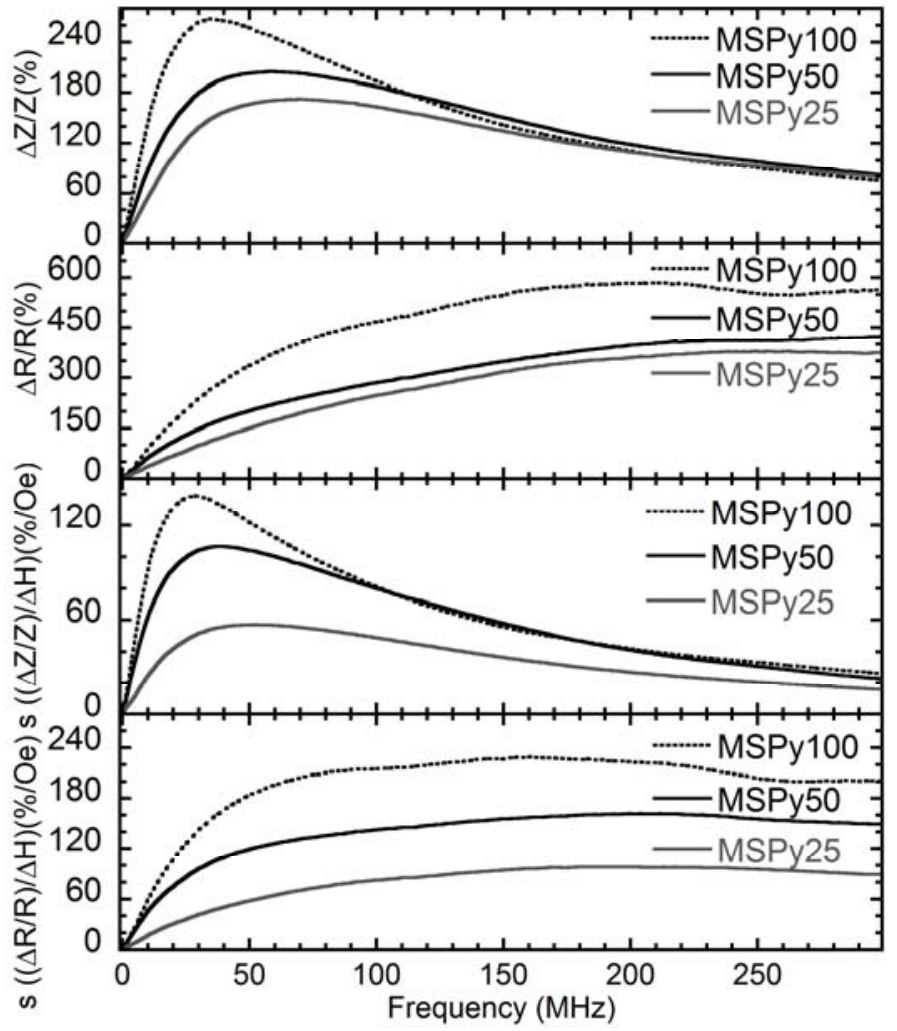

It is interesting to compare the results obtained with previous data on MI of MS structures with thicker magnetic layers and the same thickness of the $\mathrm{Cu}$ lead. A maximum MI ratio of about $270 \%$ at $35 \mathrm{MHz}$ and a maximum sensitivity of about $140 \% / \mathrm{Oe}$ at $30 \mathrm{MHz}$ are obtained for sample MSPy100. These values are larger than the ones obtained for the MS structure described in [28] where the thickness of each Py layer was $170 \mathrm{~nm}$ and the total thickness of each magnetic layer was $500 \mathrm{~nm}$. The samples were deposited in the same conditions and with the same type of MS structure (length, width and central conductor thickness) as in the present work. Despite of the lower total thickness of the ferromagnetic parts of the multilayer, sample MSPy100 has higher MI ratio and MI sensitivity to the applied field.

Finally, one can expect further increase of the MI ratio and the MI sensitivity of the MI multilayers of MSPy100, MSPy50 and MSPy25 type with an increase of the total thickness of the ferromagnetic layers to $500 \mathrm{~nm}$, i.e., matching the thickness of the central $\mathrm{Cu}$ layer, as it seems to be the best geometrical configuration leading to the highest MI results [17,31].

Of course, from the production point of view, thicker Py layers forming a multilayered structure are always an advantage due to the less number of technological steps for the deposition. Smaller number of the interfaces is also favorable because it was shown that mixed interfaces could be a reason for the decrease of the magnetic softness and the increase of anisotropy distribution width $[18,25]$. At the same time there are some important topological implications, for the case of the multilayered structures only, which have not been discussed in the literature yet. One of these topological aspects is whether 
there is an even or odd number of the Py layers in the $[\mathrm{Py} / \mathrm{Ti}]_{n}$ or $[\mathrm{Py} / \mathrm{Cu}]_{n}$ multilayered structure. The even/odd configuration can dramatically change the conditions at which the magnetic flux is closed, being especially important for miniaturized MI sensitive elements obtained by lithography. Research in this direction is being planned.

\subsection{Conclusions}

Magneto-impedance sandwiched structures consisting of a $\mathrm{Cu}$ central layer between two Py/Ti multilayered films were prepared by DC sputtering. In each structure, a different thickness of the Py layers was used $(25,50$ and $100 \mathrm{~nm})$ while maintaining the same total thickness of $300 \mathrm{~nm}$ for the magnetic material, by using the corresponding number of Py/Ti repetitions. It was found that the sandwiched structure composed of $100 \mathrm{~nm}$ thick Py layers present a better performance than the one with $50 \mathrm{~nm}$ thick Py layers and much better than the one with $25 \mathrm{~nm}$ thick Py layers. The best results obtained for the $[\mathrm{Py} / \mathrm{Ti}]_{3} / \mathrm{Cu} /[\mathrm{Ti} / \mathrm{Py}]_{3}$ sandwich structure were: $\mathrm{MI}$ ratio of $270 \%$ at $35 \mathrm{MHz}$ and a sensitivity of $140 \% / \mathrm{Oe}$ at $30 \mathrm{MHz}$. These values indicate the capabilities of optimized nanostructured multilayers to be competitive materials for small magnetic field sensitive elements well integrated into semiconductor electronics.

\section{Magnetoelastic Resonance Sensor}

The coupling between the magnetic and elastic properties of ferromagnetic materials gives rise to a number of physical effects that can be used for sensing purposes. The devices that make use of them are denominated magnetoelastic sensors. The magnetostrictive effect accounts for the change of dimensions produced in a magnetic material upon magnetization. The reverse effect, that is, the change in the magnetic state of a material caused by the application of a mechanical stress, is known as the magnetoelastic effect (also called inverse magnetostrictive or Villari effect). The magnetoelastic effect can be readily used to sense stress or any other related magnitude, and its fundamental advantage over other principles of sensing is it intrinsic non-contact nature, since magnetization changes can be detected inductively. Examples of the application of the magnetoelastic effect into successful commercial devices are described elsewhere [33].

Since in a magnetostrictive material, stress and magnetization are intimately coupled, the stress perturbation caused by a sound wave traveling in the material is accompanied by a magnetization one, giving rise to a magnetoelastic wave, whose mathematical formalism is treated exhaustively in [34]. Under adequate circumstances, the traveling magnetoelastic waves become stationary, giving rise to resonances. For example, when a magnetic sample of length $L$ is magnetized by an alternating magnetic field, if the wavelength of the magnetoelastically induced sound wave matches the dimension of the sample, a resonance is built up, which is called magnetoelastic resonance. For a sample of density $\rho$, having a Young modulus $E$, oscillating with free ends, the resonant frequency is given by:

$$
f_{r}=\frac{1}{2 L} \sqrt{\frac{E}{\rho}}
$$

At resonance, great magnetization changes takes places and the permeability of the sample, that can be detected inductively using a coil wounded around the sample, increases greatly. Figure 7 displays 
a typical magnetoelastic resonance measured in a commercial amorphous ribbon $5 \mathrm{~cm}$ long. It is especially important to state that, due to the magnetoelastic coupling, the value of the Young's modulus $E$ depends on the magnetic state of the sample (this is the so-called $\Delta E$ effect). Therefore, the frequency of resonance can be tuned by biasing the sample by a suitable magnetic field as exemplified in the inset of Figure 7.

Figure 7. Magnetoelastic resonance of an amorphous ribbon. The inset shows the dependence of the resonant frequency on the applied magnetic field.

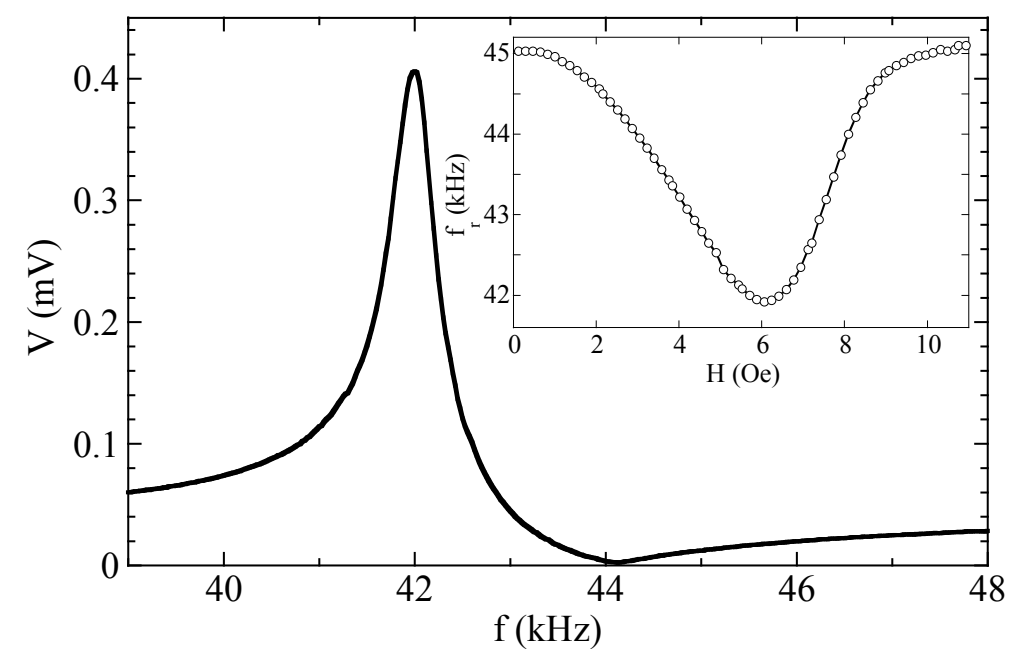

With applications in mind, the magnetoelastic resonance can be used to remotely detect any parameter that affects the magnetostrictive element, such as temperature [35], or pressure [36]. The magnetoelastic material can be also covered by a chemically responsive layer to develop gas, humidity or $\mathrm{pH}$ sensors [37]. Probably the most successful application of the magnetoelastic resonance are the acousto-magnetic antishoplifting labels, whose principle of operation is described in [33]. In this work we describe the use of the magnetoelastic resonance to determine the viscosity of lubricant oils, through the damping caused by the oil in the oscillations of a magnetoelastic sample.

\subsection{Experimental: Oil Viscosity Sensor}

The roles of lubricant oils in machinery include not only friction reduction, but also refrigeration, segregation of contaminants and debris and protection against wear and corrosion. These tasks degrade the oil, so it must be replaced to prevent failures. For optimum performance, it is necessary to develop sensors that provide online and real-time measurements of the condition of the lubricant oil. The viscosity of the oil is severely affected by the degradation of the oil due to aging and/or contamination and, therefore, a sensor determining continuously the viscosity of the lubricant oil can help implementing a predictive maintenance strategy in machinery.

The amplitude and frequency of the magnetoelastic response of a material immersed in a viscous medium depends on the viscosity of such medium. Our prototype uses a commercial amorphous ribbon (VITROVAC 4040 kindly provided by Vacuumschmelze GmbH, Hanau, Germany), 33 mm long, $6 \mathrm{~mm}$ wide and $23 \mu \mathrm{m}$ thick. One extreme of the sample is glued to a glass rod and inserted in the oil under test, which fills a $4 \mathrm{~mL}$ vial (see Figure 8). For detecting the resonance, a two-coil system with 
separated excitation and detection coils is used. The detection coil is complemented with a compensation coil connected in opposition. The bias field to set the optimum magnetic state of the sample is provided by a pair of Helmholtz coils. The resonance measurements were performed using a spectrum analyzer performing a continuous frequency sweep.

Figure 8. (a) The magnetoelastic sample is glued to a glass rod, fixed to the cap of the vial;

(b) When the cap is fitted to the vial, the sample is immersed in the oil.

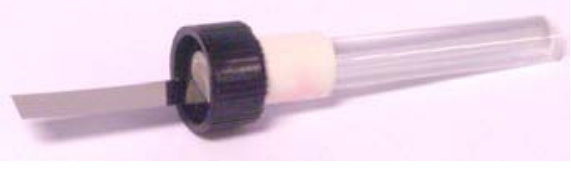

(a)

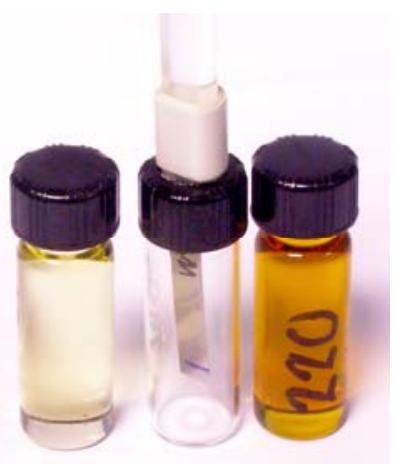

(b)

Oils from two different families covering a wide range of viscosities were used for the experiments. Three samples of oil used in hydraulic systems had viscosities of 32.4, 67.1 and 108.2 cSt respectively. Two additional samples of high duty oils used in gearboxes had viscosities 218.2 and $325.9 \mathrm{cSt}$ respectively. The viscosity of all the samples was determined using the ASTM D-445 procedure (American Society for Testing and Materials, ASTM International, West Conshohocken, PA, USA), within an accuracy of $0.1 \mathrm{cSt}$ [38].

\subsection{Data Analysis Procedure}

To analyze the data we have developed a phenomenological approach to describe the frequency response of a magnetoelastic material. The magnetoelastic resonance is characterized by a sharp maximum at the resonance followed by a null minimum at the antiresonance (see Figure 7). Using the formalism of linear systems, an analytical expression for the transference function can be readily written: the resonance imposes a couple of complex conjugated poles in the denominator, and the antiresonance is described with a couple of complex conjugated zeros in the numerator. The transference function is then:

$$
G(s)=\frac{\omega_{r}^{2}}{\omega_{a}^{2}} \cdot \frac{s^{2}+2 \delta_{a} \omega_{a} s+\omega_{a}^{2}}{s^{2}+2 \delta_{r} \omega_{r} s+\omega_{r}^{2}}
$$

where $\omega_{r}$ is the value of the resonance frequency, $\omega_{a}$ the antiresonance, and $\delta_{a}$ and $\delta_{r}$ are damping parameters. In this formalism $s=j \omega$, where $\omega$ is a real frequency and $j=\sqrt{-1}$.

Experimentally, the measured resonance curves can be fitted to:

$$
V(\omega)=A\left|\frac{\omega^{2}-2 j \delta_{a} \omega_{a} \omega-\omega_{a}^{2}}{\omega^{2}-2 j \delta_{r} \omega_{r} \omega-\omega_{r}^{2}}\right|+a \omega+b
$$


The only parameters to be fitted are the resonance $\omega_{r}=2 \pi f_{r}$ and the antiresonance $\omega_{a}=2 \pi f_{a}$ frequencies, and the damping constants $\delta_{r}$ and $\delta_{a}$, together with the parameters $A, a$, and $b$ that account for the amplitude and a linear background respectively. Figure 9 shows the excellent agreement found by a least squares fit for the resonance curve measured in a $37 \mathrm{~mm}$ long, $6 \mathrm{~mm}$ wide VITROVAC ribbon.

Figure 9. Fitting of a measured resonance curve to Equation (3). Best fit parameters are: $f_{r}=56.73 \mathrm{kHz} ; \delta_{r}=0.0022 ; f_{a}=60.82 \mathrm{kHz} ; \delta_{a}=0.0121 ; A=38.07 \mathrm{mV}$; $2 \pi a=8.57 \times 10^{-6} \mathrm{mV} / \mathrm{Hz} ; b=-55.97 \mathrm{mV}$.

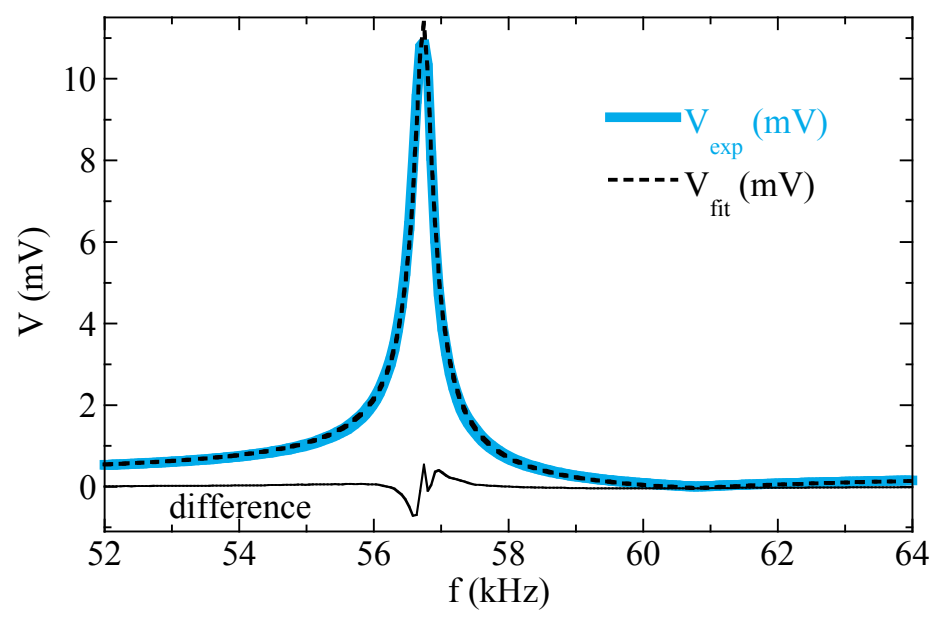

\subsection{Results and Discussion}

Figure 10 shows the resonance curves obtained with the magnetoelastic material immersed in oils with different viscosities. For better comparison, the linear background has been subtracted from the measured curves. All the curves were obtained at room temperature with an applied bias magnetic field of $6.7 \mathrm{Oe}$, which was checked to provide the maximum amplitude of the resonance in air. The AC excitation amplitude was set to about $0.1 \mathrm{Oe}$, which is relatively large to obtain a good signal to noise response, but still low to avoid non-linear effects [39].

As expected, the amplitude of the resonance curves systematically decreases as the viscosity of the oil increases, whereas the width of the resonance increases with the viscosity, due to the increasing damping. The resonance frequency and the amplitude can be extracted directly from the measured curves to obtain its dependence on viscosity [40]. Additionally, Figure 10 shows the fits of the data to Equation (1). The fitting results allow us to accurately determine these parameters, but also the damping parameter $\delta_{r}$ intimately related to the viscosity.

Figure 11 represents the results obtained from the fittings. Figure 11c shows that the frequency shift with respect to the resonance in air follows a linear relation with the square root of the product of the viscosity and the density of the oils, as predicted by the theory for low viscosity regimens [41]. The dependence of the damping parameter $\delta_{r}$ on the viscosity reveals two distinct slopes that correlate with the different nature of the oils under test. This is an indication of the capabilities of the method for detecting fine features in the viscosity of the oils. 
Figure 10. Magnetoelastic resonance curves measured for oils with different viscosities. The dashed lines correspond to the fitting of the experimental curves to Equation (2).

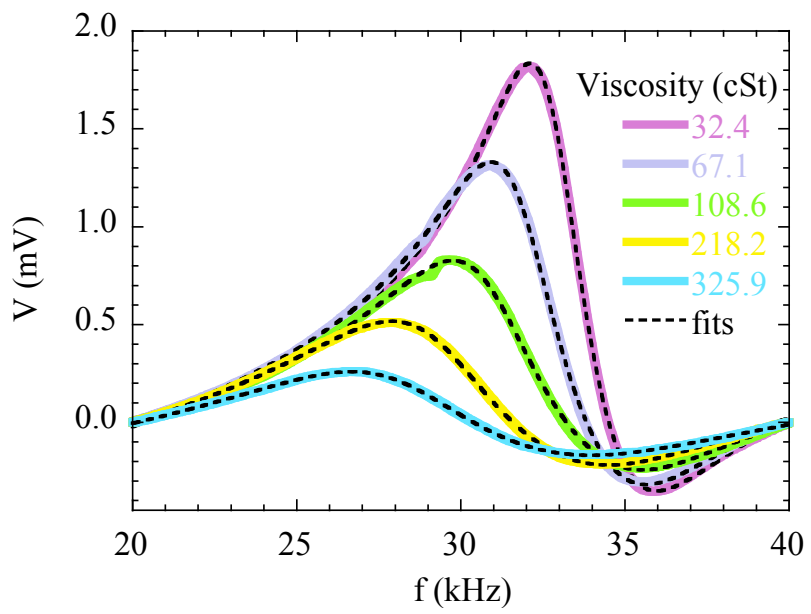

Figure 11. Dependence of best-fit parameters on oil viscosity $\eta$. (a) Amplitude of the resonance; (b) Resonance frequency; (c) The resonance frequency shift (from the resonance frequency in air) scales with the square root of the product of the viscosity times the density of the oil; (d) Damping parameter.
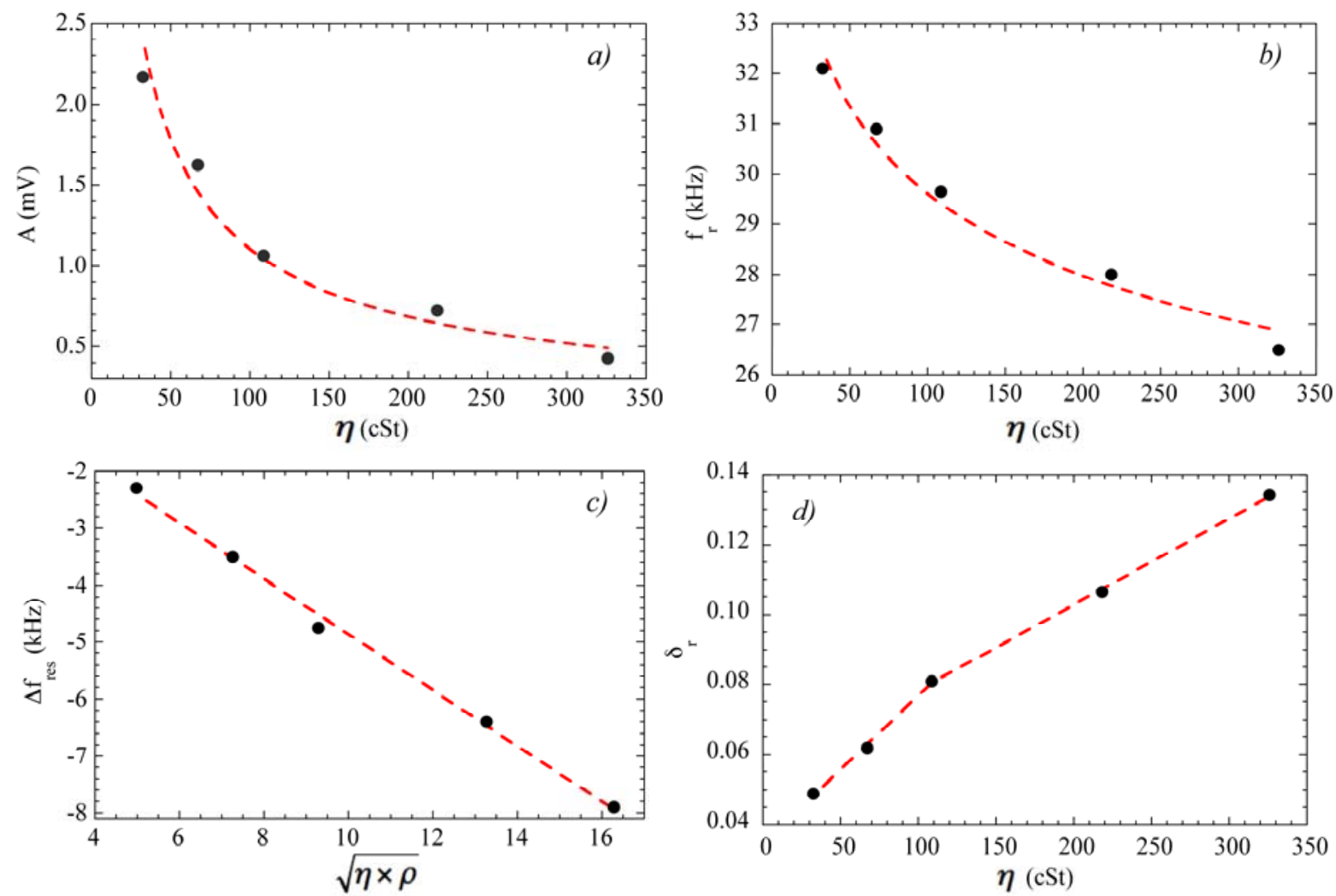

\subsection{Conclusions and Outlook}

The magnetoelastic resonance is revealed as a useful technique to determine the viscosity of lubricant oils in a wide range of viscosities, from 32 to $326 \mathrm{cSt}$. The fitting of the resonance curve to a phenomenological equation allows determining not only the dependence of the resonance frequency 
and the amplitude of the resonance on the viscosity, but also the damping parameter. Any of these parameters can be used for designing a sensor for on-line and real-time assessing the degradation of lubricant oils based on viscosity measurements. Further development will attempt to determine the viscosity from the time-dependent attenuation of the oscillations of the magnetoelastic samples after a pulsed excitation at the resonant frequency.

\section{Magnetoelectric Sensor}

The combination of magnetostrictive amorphous ferromagnetic ribbons with piezoelectric materials, allows obtaining magnetoelectric laminated composites, that show an extremely high sensitivity for magnetic field detection. Combinations of VITROVAC type magnetic alloys epoxyed to Polyvinylidene Fluoride (PVDF) piezoelectric polymer give as result magnetoelectric coefficients above $80 \mathrm{~V} / \mathrm{cm}$ Oe. Also, high temperature new piezopolymers as polyimides are can be used for the magnetoelectric detection at temperatures as high as $100{ }^{\circ} \mathrm{C}$.

\subsection{Magnetoelectric Experimental Details}

We construct magnetoelectric three-layer sandwich-like with longitudinal magnetostrictive operation and transverse piezoelectric response (L-T type) laminated composites by gluing two equal magnetostrictive ribbons to opposite sides of polymer piezoelectric films with an adhesive epoxy resin (Figure 12, upper left). Magnetostrictive ribbons belonging to the family of Fe-Co-Ni-Si-B, Fe-rich metallic glasses have a measured magnetostriction that ranges between $\lambda_{s} \approx 8-30 \mathrm{ppm}$ and maximum value for the piezomagnetic coefficient $d_{33}=d \lambda / d H$ of about $0.6-1.5 \times 10^{-6} / \mathrm{Oe}$. This last parameter will modulate the magnetoelectric response of the composite as a function of the applied bias magnetic field.

Concerning the piezoelectric material we firstly used the well-known polymer PVDF, the well-known piezoelectric polymer [42], with glass transition and melting temperatures about $-35{ }^{\circ} \mathrm{C}$ and $171^{\circ} \mathrm{C}$, respectively, but a Curie temperature of $\approx 100{ }^{\circ} \mathrm{C}$. This makes its piezoelectric response to decay quickly above $70{ }^{\circ} \mathrm{C}$ (Figure 12). To develop a ME device being able to operate at higher temperatures, we have also tested new amorphous piezoelectric polymers of the family of the polyimides. Detailed information about synthesis, thermal characterization and electric polarization processes can be found in [43]. We only will remark briefly that its main parameters are a glass transition temperature of $T_{\mathrm{g}} \approx 200{ }^{\circ} \mathrm{C}$ [44] and a degradation temperature of $T_{\mathrm{d}} \approx 510{ }^{\circ} \mathrm{C}$, temperatures that make these polyimides suitable for our purposes.

Taking advantage of the magnetoelastic resonance effect that enhances the magnetostrictive response, all measurements have been taken at resonance. For that, we first determine the static magnetic field $H_{\mathrm{DC}}$ necessary to induce the maximum amplitude of that resonance, using the measurement set-up previously described. Then, we measure the induced magnetoelectric voltage $V_{\mathrm{ME}}$ in the sandwich laminate (through two small silver ink contacts located at both opposite magnetostrictive ribbons) by the following procedure: under a $H_{\mathrm{AC}}$ magnetic excitation applied along the length axis, the magnetostrictive ribbons will elongate and shrink along the same direction. This will make the piezoelectric polymer film to undergo an AC longitudinal strain, inducing a dielectric polarization change in its transverse direction (L-T operation). 
Figure 12. Measured remnant polarization as a function of temperature for commercial PVDF piezoelectric polymer and the new piezolelectric 2,6( $\beta-\mathrm{CN}) \mathrm{APB} / \mathrm{ODPA}$ (poly-2,6) polyimide.

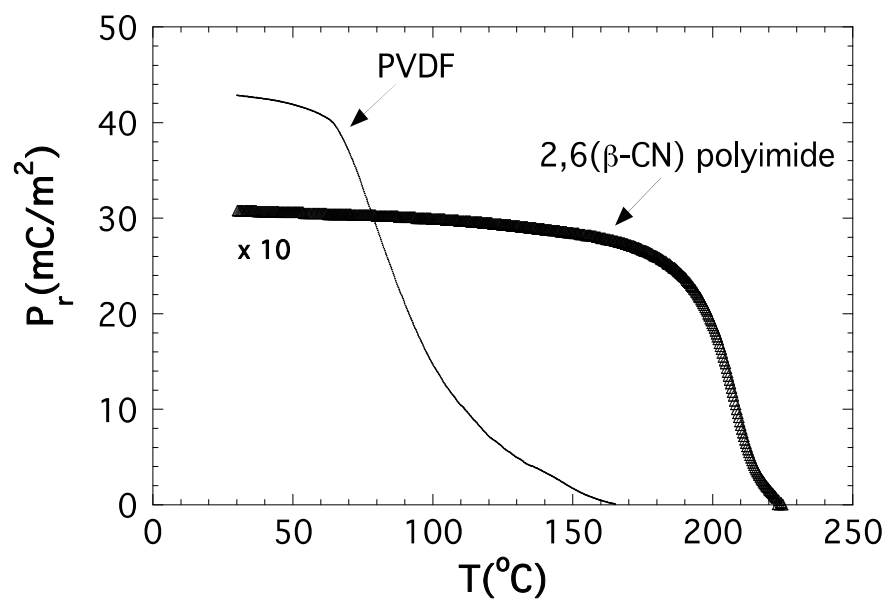

Thus, we can determine simultaneously (a) the magnetoelectric response dependence as the bias field $H_{\mathrm{DC}}$ changes; and (b) at the $H_{\mathrm{DC}}$ value for the maximum magnetoelastic resonance amplitude, the magnetoelectric voltage dependence versus the applied $H_{\mathrm{AC}}$ magnetic excitation, that is the so called sensitivity of the magnetoelectric composite.

From the measured induced magnetoelectric voltage, the magnetoelectric coefficient $\alpha_{\mathrm{ME}}$ can be directly derived as [45]:

$$
\alpha_{M E}=\frac{d E_{1}}{d H_{3}}=\frac{1}{t}\left(\frac{\delta V_{M E}}{\delta H_{a c}}\right)
$$

In this equation, $t$ is the thickness of the piezoelectric layer. For L-T operation (Figure 13) this coefficient can also be written as $\alpha_{\mathrm{ME}}=\alpha_{31}$.

Figure 13. Geometry of the three-layer L-T sandwich configuration: magnetostrictive ribbons are longitudinally magnetized while the piezoelectric polymers were transversely poled.

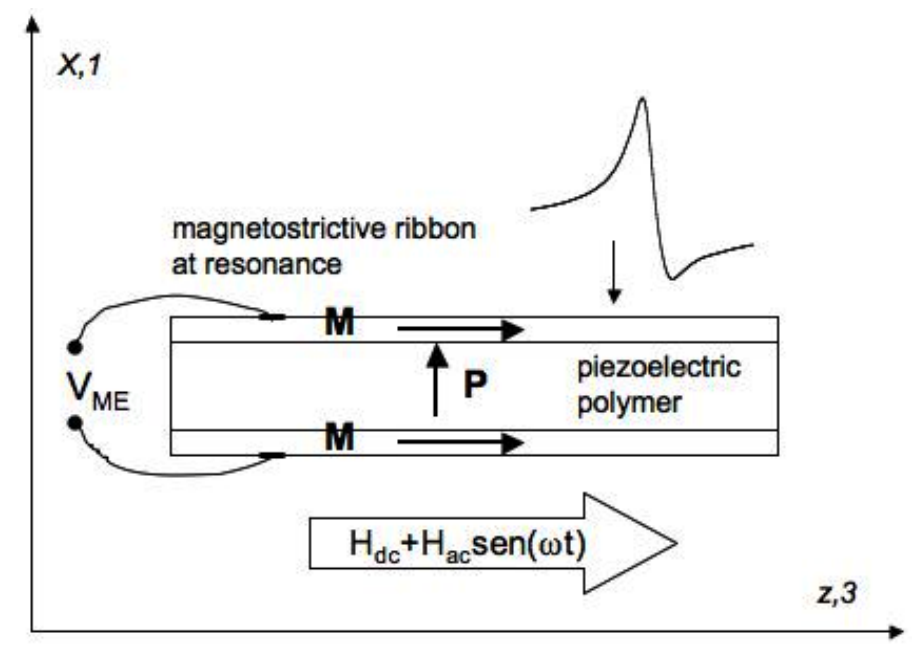




\subsection{Applications Based on the Magnetoelectric Effect}

Applications based on the ME effect are as widespread as magnetic field sensors [46,47], current sensors [48], transformers [49], microwave devices [50] and FMR resonators and filters [51,52]. The highest ME response has been reported for laminated magnetostrictive/piezoelectric polymer composites. Fang et al. [53] reached a magnetoelectric voltage coefficient of $21.46 \mathrm{~V} \mathrm{~cm}^{-1} \mathrm{Oe}^{-1}$ for a METGLAS 2605 SA1/PVDF (Metglas, Conway, SC, USA) laminate achieved at non-resonance frequencies and is, so far, the highest response obtained at sub-resonance frequencies. At the longitudinal Magnetoelastic Resonance (MER) of the magnetostrictive constituent, energy transference from magnetic to elastic, and vice versa, is maximum. This energy conversion at the resonance turns out to be very sharp for ME laminates, while frequency bandwidth for applications based in this MER enhancement effect remains limited. Jin et al. [54] reached a magnetoelectric voltage coefficient of $383 \mathrm{~V} \mathrm{~cm}^{-1} \mathrm{Oe}^{-1}$ on cross-linked P(VDF-TrFE)/METGLAS $2605 \mathrm{SA} 1$, the highest reported to date.

Our first measurements have been performed with laminated composites of the type VITROVAC $4040^{\circledR} / \mathrm{PZT}$ or PVDF/VITROVAC $4040^{\circledR}$. As can be seen in Figure 14 both type of laminates show a good magnetoelectric response (of $125 \mathrm{~V} \mathrm{~cm}^{-1} \mathrm{Oe}^{-1}$ and $80 \mathrm{~V} \mathrm{~cm}^{-1} \mathrm{Oe}^{-1}$ for Lead zirconium titanate (PZT) and PVDF containing laminates, respectively) at low applied magnetic fields (about 10 Oe, due to the anisotropy field of the VITROVAC ribbon).

We have also measured the sensitivity of the VITROVAC $4040^{\circledR} /$ PVDF/VITROVAC $4040^{\circledR}$ device as a function of temperature, getting a decrease from $357 \mathrm{mV} / \mathrm{Oe}$ (sensitivity below $0.3 \mathrm{nT}$ ) at room temperature to $142 \mathrm{mV} / \mathrm{Oe}$ at $80{ }^{\circ} \mathrm{C}$ (sensitivity below $0.8 \mathrm{nT}$ ).

In order to avoid this observed sensitivity decrease when increasing temperature, we have fabricated the same L-T structured magnetoelectric laminates with the same magnetostrictive constituents but using a 40/60 copolyimide as high temperature piezoelectric constituent [55].

Figure 14. Measured $\alpha_{\mathrm{ME}}$ magnetoelectric coupling coefficient for our L-T type laminated composite with VITROVAC $4040^{\circledR}$ as magnetostrictive constituent and ceramic PZT or PVDF polymer as piezoelectric constituent. Sizes of the devices are almost the same, as hinted by their close resonant frequencies.

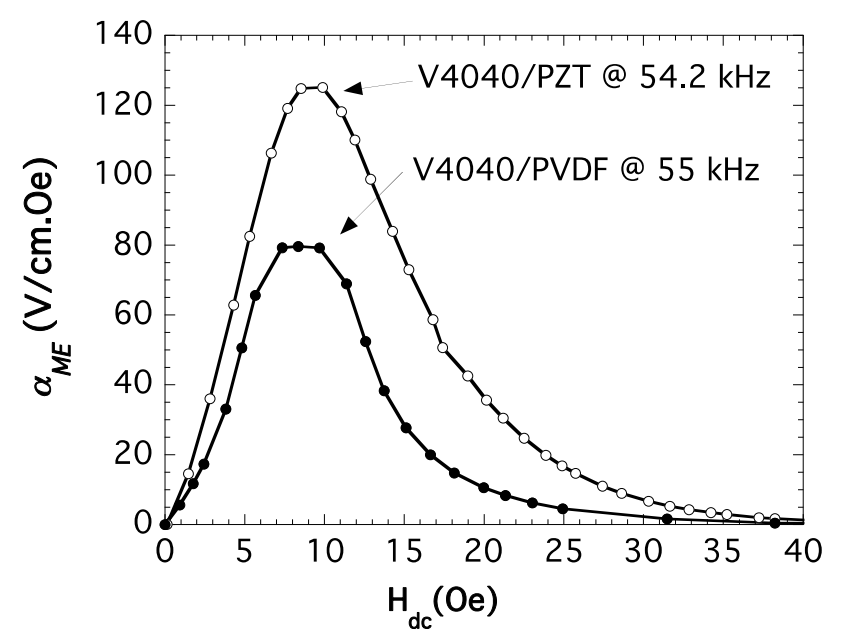


As it can be seen in Figure 15 (Reprinted with permission from [56]. Copyright 2013 IEEE Xplore), even if the magnetoelectric response turns out to be much more modest than when using PVDF piezopolymer, the great advantage is that sensitivity keeps constant up to high temperatures $>80{ }^{\circ} \mathrm{C}$, with values about $37 \mathrm{mV} / \mathrm{Oe}$ (sensitivity below $2.7 \mathrm{nT}$ ).

Figure 15. Room temperature measured $\alpha_{\mathrm{ME}}$ magnetoelectric coupling coefficient for our L-T type laminated composite with METGLAS $2826 \mathrm{MB}$ as magnetostrictive constituent and a 40/60 copolyimide as piezoelectric constituent. Reprinted with permission from [56]. Copyright 2013 IEEE Xplore.

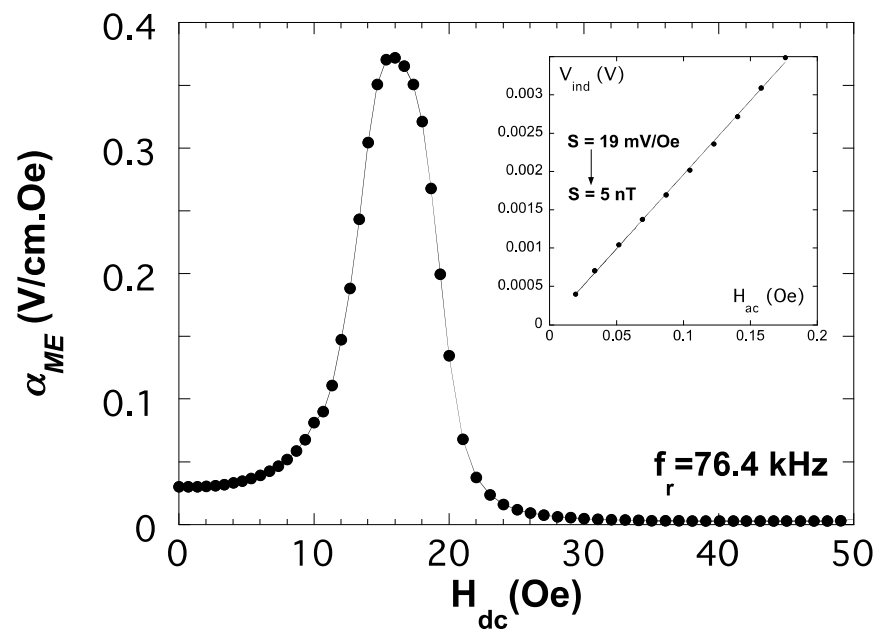

Efforts to get wider bandwidths for MER and ME applications have been mainly based on magnetic field tuning procedures either in bimorph or tri-layered structures, but the maximum achieved frequency of operation has been some tenths of $\mathrm{kHz}$ [57]. Another way to get high frequencies of operation can be based on the relationship between length and resonant frequency value $\left(f_{r} \propto 1 / L\right)$ of magnetostrictive ribbons at the magnetoelastic resonance. So, our efforts are now focused on fabricating short magnetoelectric L-T type laminates showing good magnetoelectric response at high frequencies. Nevertheless, the higher the resonant frequency the lowest the amplitude of the resonance and as a first consequence, the magnetoelectric response will be also decreased. It is clear that a compromise between length of the device and so working frequency, and induced magnetoelectric signal, must be achieved.

Thus, we have developed a device $1 \mathrm{~cm}$ long for which the resonant (working) frequency rises to $230 \mathrm{kHz}$ (See Figure 16), and the measured magnetoelectric coupling coefficient is about $15 \mathrm{~V} \mathrm{~cm}^{-1}$ $\mathrm{Oe}^{-1}$ when PVDF is used as piezoelectric constituent. Following this line of reasoning, we expect to construct a $0.5 \mathrm{~cm}$ long device that will work at a resonant frequency about $500 \mathrm{kHz}$. This fact, combined with the use of a high temperature piezopolymer as the polyimides previously described, can lead to a very useful class of magnetoelectric laminates working simultaneously at high temperature and within the radiofrequency range, both characteristics of great interest for low distance near field communications in aggressive environments (i.e., the desert, a tunnel or fighting a fire). 
Figure 16. Magnetoelastic resonance curves measured for different ribbon pieces with length ranging from $4 \mathrm{~cm}$ to $1 \mathrm{~cm}$. The inset shows also the measured resonance frequency and the induced signal at the resonance, as a function of the length of the resonant magnetostrictive ribbon.

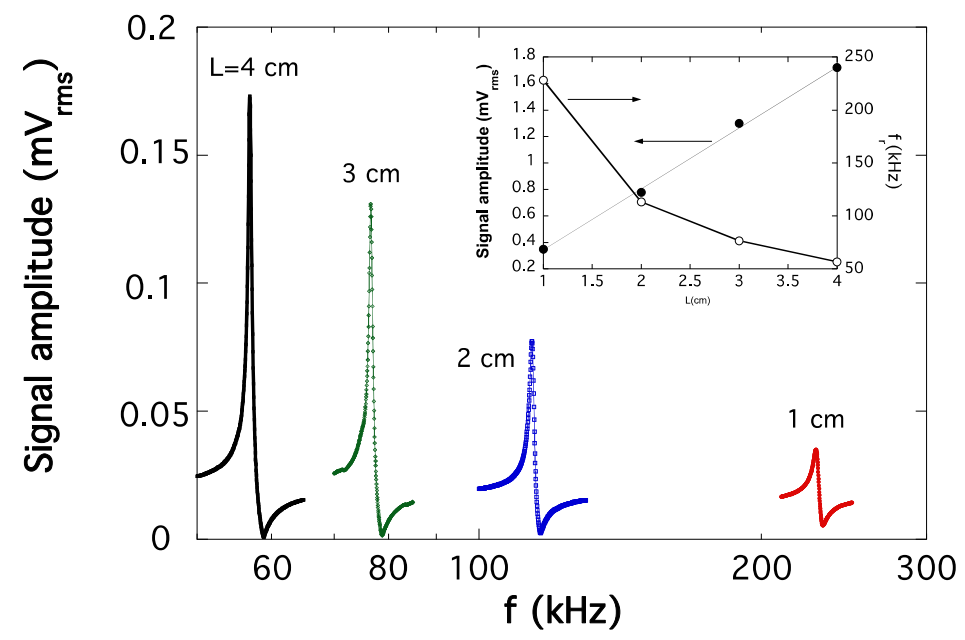

\subsection{Conclusions}

Combining the excellent magnetoelastic response of magnetostrictive amorphous ferromagnetic ribbons with piezoelectric polymers, we have fabricated short length magnetoelectric laminated composites that show an extremely high sensitivity for magnetic field detection. We have already fabricated devices that work within the radiofrequency range and we expect to use them with high temperature new piezopolymers (polyimides) in order to achieve magnetoelectric detection at temperatures as high as $100{ }^{\circ} \mathrm{C}$.

\section{Conclusions/Outlook}

Our recent research results on sensor technologies based on soft magnetic materials have been reviewed. First, the effect of the thickness of Py layers in multilayer sandwiched magneto-impedance structures has been studied. Second, the magnetoelastic resonance has been shown to produce useful results to determine the viscosity of lubricant oils, based on a new technique for analyzing the resonance curves. Finally, new magnetoelectric composites with excellent performance for low magnetic field detection have been described. The results presented in each technology constitute single steps in a long range research strategy of the Group of Magnetism and Magnetic Materials of the University of the Basque Country in Spain, aimed to develop high performance sensing devices based on soft magnetic materials.

\section{Acknowledgments}

The authors would like to thank the financial support from the Basque Government under ACTIMAT (Etortek program), PE12UN025 (Saiotek program) and IT711-13 (Research Groups) projects; and from the Spanish Government under project MAT2011-27573-C04. Andoni Lasheras 
wants to thank the Basque Government for financial support under PhD Grant. Technical and human support provided by SGIker (UPV/EHU), especially by Iñaki Orue, is gratefully acknowledged.

\section{Author Contributions}

Andrey Svalov fabricated the magneto-impedance nanostructures deposited and the magnetic characterization of the samples. David de Cos designed the GMI measurement setup, and Eduardo Fernández performed the measurements and interpreted the data of the magneto-impedance measurements. Galina V. Kurlyandskaya and Eduardo Fernández wrote the part of the manuscript related with the magneto-impedance Sensor. Iñaki Bravo-Imaz performed the measurements and interpreted the data of the Magnetoelastic Resonance Sensor measurements. Alfredo García-Arribas wrote the part of the manuscript related with the Magnetoelastic Resonance Sensor. Andoni Lasheras performed the measurements and interpreted the data of the Magnetoelectric Sensor measurements. Jon Gutiérrez wrote the part of the manuscript related with the Magnetoelectric Sensor. José M. Barandiarán wrote the introduction and conclusions of the manuscript. All authors discussed the results and implications, and commented on the manuscript at all stages. All authors read and approved the final manuscript.

\section{Conflicts of Interest}

The authors declare no conflict of interest.

\section{References}

1. Prinz, G.A. Magnetoelectronics applications. J. Magn. Magn. Mater. 1999, 200, 57-68.

2. Ripka, P.; Zaveta, K. Magnetic Sensors: Principles and Applications. In Handbook of Magnetic Materials; Buschow, K.H.J., Ed.; Elsevier: Amsterdam, The Netherlands, 2009; Volume 18, pp. 347-420.

3. Kurlyandskaya, G.V.; de Cos, D.; Volchkov, S.O. Magnetosensitive Transducers for Nondestructive Testing Operating on the Basis of the Giant Magnetoimpedance Effect: A Review. Russ. J. Nondestruct. Test. 2009, 45, 377-398.

4. Mahdi, A.E.; Panina, L.; Mapps, D. Some new horizons in magnetic sensing: High-Tc SQUIDs, GMR and GMI materials. Sens. Actuators A 2003, 105, 271-285.

5. Koch, H. Magnetic Sensors; VCH Verlagssgesellschaft: Weinheim, Germany, 1989.

6. Makhotkin, V.E.; Shurukhin, B.P.; Lopatin, V.A.; Marchukov, P.Y.; Levin, Y.K. Magnetic Field Sensors Based on Amorphous Ribbons. Sens. Actuators A Phys. 1991, 27, 759-762.

7. Reig, C.; Cubells-Beltran, M.-D.; Ramirez Munoz, D. Magnetic Field Sensors Based on Giant Magnetoresistance (GMR) Technology: Applications in Electrical Current Sensing. Sensors 2009, 9, 7919-7942.

8. Nishibe, Y.; Yamadera, H.; Ohta, N.; Tsukada, K.; Nonomura, Y. Thin Film Magnetic Field Sensor Utilizing Magneto Impedance Effect. Sens. Actuators A Phys. 2000, 82, 155-160. 
9. García-Arribas, A.; Martínez, F.; Fernández, E.; Ozaeta, I.; Kurlyandskaya, G.V.; Svalov, A.V.; Berganzo, J.; Barandiaran, J.M. GMI detection of magnetic-particle concentration in continuous flow. Sens. Actuators A Phys. 2011, 172, 103-108.

10. Fernandez, E.; Garcia-Arribas, A.; Volchkov, S.O.; Kurlyandskaya, G.V.; Barandiaran, J.M. Differences in the magneto-impedance of $\mathrm{FeNi} / \mathrm{Cu} / \mathrm{FeNi}$ multilayers with open and closed magnetic path. IEEE Trans. Magn. 2010, 46, 658-661.

11. Correa, M.A.; Bohn, F.; Chesman, C.; da Silva, R.B.; Viegas, A.D.C.; Sommer, R.L. Tailoring the magnetoimpedance effect of NiFe/Ag multilayer. J. Phys. D Appl. Phys. 2010, 43, doi:10.1088/0022-3727/43/29/295004.

12. Knobel, M.; Vázquez, M.; Kraus, L. Giant Magnetoimpedance. In Handbook of Magnetic Materials; Buschow, K.H.J., Ed.; Elsevier: Amsterdam, The Netherlands, 2003; Volume 5, pp. 497-563.

13. Tannous, C.; Gieraltowski, J. Giant magnetoimpedance and its applications. J. Mater. Sci. Mater. Electron. 2004, 15, 125-133.

14. Phan, M.H.; Peng, H.X. Giant magnetoimpedance materials: Fundamentals and applications. Prog. Mater. Sci. 2008, 53, 323-420.

15. Kurlyandskaya, G.V.; Muñoz, J.L.; Barandiaran, J.M.; García-Arribas, A.; Svalov, A.V.; Vas'kovskiy, V.O. Magnetoimpedance of sandwiched films: Experimental results and numerical calculations. J. Magn. Magn. Mater. 2002, 242-245, 291-293.

16. Gardner, D.S.; Schrom, G.; Paillet, F.; Jamieson, B.; Karnik, T.; Borkar, S. Review of on-chip inductor structures with magnetic films. IEEE Trans. Magn. 2009, 45, 4760-4766.

17. Panina, L.V.; Mohri, K. Magneto-impedance in multilayer films. Sens. Actuators A Phys. 2000, $81,71-77$.

18. Kurlyandskaya, G.V.; García-Arribas, A.; Fernández, E.; Svalov, A.V. Nanostructured Magnetoimpedance Multilayers. IEEE Trans. Magn. 2012, 48, 1375-1380.

19. De Cos, D.; Fry, N.; Orue, I.; Panina, L.V.; García-Arribas, A.; Barandiaran, J.M. Very large magnetoimpedance (MI) in FeNi/Au multilayer film systems. Sens. Actuators A Phys. 2005, 129, 256-259.

20. Sugita, Y.; Fujiwara, H.; Sato, T. Critical thickness and perpendicular anisotropy of evaporated permalloy films with stripe domains. Appl. Phys. Lett. 1967, 10, 229-231.

21. Svalov, A.V.; Aseguinolaza, I.R.; Garcia-Arribas, A.; Orue, I.; Barandiaran, J.M.; Alonso, J.; Fernandez-Gubieda, M.L.; Kurlyandskaya, G.V. Structure and magnetic properties of thin permalloy films near the "transcritical" state. IEEE Trans. Magn. 2010, 46, 333-336.

22. Volchkov, S.O.; Fernandez, E.; Garcia-Arribas, A.; Barandiaran, J.M.; Lepalovskij, V.N.; Kurlyandskaya, G.V. Magnetic Properties and Giant Magnetoimpedance of FeNi-Based Nanostructured Multilayers With Variable Thickness of the Central Cu Lead. IEEE Trans. Magn. 2011, 47, 3328-3331.

23. Svalov, A.V.; Fernandez, E.; Garcia-Arribas, A.; Alonso, J.; Fdez-Gubieda, M.L.; Kurlyandskaya, G.V. FeNi-based magnetoimpedance multilayers: Tailoring of the softness by magnetic spacers. Appl. Phys. Lett. 2012, 100, doi:10.1063/1.4704984. 
24. Vas'kovskii, V.O.; Savin, P.A.; Volchkov, S.O.; Lepalovskii, V.N.; Bukreev, D.A.; Buchkevich, A.A. Nanostructuring effects in soft magnetic films and film elements with magnetic impedance. Tech. Phys. 2013, 58, 105-110.

25. Alzola, N.V.; Kurlyandskaya, G.V.; Larrañaga, A.; Svalov, A.V. Structural Peculiarities and Magnetic Properties of FeNi Films and FeNi/Ti-Based Magnetic Nanostructures. IEEE Trans. Magn. 2012, 48, 1605-1608.

26. Muñoz, J.L.; Kurlyandskaya, G.V.; Barandiaran, J.M.; Potapov, A.P.; Lukshina, V.A.; Vazquez, M. Nonuniform anisotropy and magnetoimpedance in stress annealed amorphous ribbons. Phys. Met. Metall. 2001, 91, 139-142.

27. Garcia-Arribas, A.; Barandiaran, J.M.; Herzer, G. Anisotropy field distribution in amorphous ferromagnetic alloys from second harmonic response. J. Appl. Phys. 1992, 71, doi:10.1063/1.350992.

28. De Cos, D.; García-Arribas, A.; Barandiaran, J.M. Analysis of magnetoimpedance at high frequency using a microstrip transmission line. Sens. Actuators A. Phys. 2004, 115, 368-375.

29. Barandiarán, J.M.; García-Arribas, A.; de Cos, D. Transition from quasistatic to ferromagnetic resonance regime in giant magnetoimpedance. J. Appl. Phys. 2006, 99, doi:10.1063/1.2195898.

30. Fernández, E.; Svalov, A.V.; Kurlyandskaya, G.V.; Garcia-Arribas, A. GMI in Nanostructured FeNi/Ti Multilayers With Different Thicknesses of the Magnetic Layers. IEEE Trans. Magn. 2013, 49, 18-21.

31. Fernández, E.; Svalov, A.V.; García-Arribas, A.; Feuchtwanger, J.; Barandiaran, J.M.; Kurlyandskaya, G.V. High Performance Magnetoimpedance in FeNi/Ti Nanostructured Multilayers with Opened Magnetic Flux. J. Nanosci. Nanotechnol. 2012, 12, 7496-7500.

32. Garcia-Arribas, A.; Fernandez, E.; Svalov, A.V.; Kurlyandskaya, G.V.; Barrainkua, A.; Navas, D.; Barandiaran, J.M. Tailoring the magnetic anisotropy of thin film permalloy microstrips by combined shape and induced anisotropies. Eur. Phys. J. B 2013, 86, doi:10.1140/epjb/e201330933-6.

33. García-Arribas, A.; Barandiaran, J.M.; Gutierrez, J. Magnetoelastic Sensors. In Encyclopedia of Sensors; Grimes, C.A., Dickey, E.C., Eds.; American Scientific Publishers: Valencia, CA, USA, 2005; Volume 5, p. 467.

34. Du Tremolet de Laichesserie, E. Magnetostriction, Theory and Application of Magnetoelasticity; CRC Press: Boca Raton, FL, USA, 1993.

35. García-Arribas, A.; de Cos, D.; Gutiérrez, J.; Barandiaran, J.M. Selectable temperature sensitivity of the magnetoelastic resonance. Sens. Actuators A Phys. 2003, 106, 111-116.

36. Jain, M.K.; Grimes, C.A. A wireless magnetoelastic micro-sensor array for simultaneous measurements of temperature and pressure. IEEE Trans. Magn. 2001, 37, 2022-2024.

37. Grimes, C.A.; Mungle, C.S.; Zeng, K.; Jain, M.K.; Dreschel, W.R.; Paulose, M.; Ong, K.G. Wireless magnetoelastic resonance sensors: A critical review. Sensors 2002, 2, 294-313.

38. ASTM International. Available online: http://www.astm.org/Standards/D445.htm (accessed on 14 January 2014).

39. García-Arribas, A.; Barandiaran, J.M.; Gutiérrez, J.; Sagastabeitia, I. On the origin of the nonlinear and chaotic behavior of the magnetoelastic resonance. J. Appl. Phys. 1997, 81, 5686-5688. 
40. Bravo-Imaz, I.; García-Arribas, A.; Gorritxategi, E.; Arnaiz, A.; Barandiaran, J.M. Magnetoelastic viscosity sensor for on-line status assement of lubricant oils. IEEE Trans. Magn. 2013, 49, 113-116.

41. Stoyanov, P.G.; Grimes, C.A. A remote query magnetostrictive viscosity sensor. Sens. Actuators A Phys. 2000, 80, 8-14.

42. Kawai, H. The piezoelectricity of Poli (vinylidene Fluoride). Jpn. J. Appl. Phys. 1969, 8, 975-976.

43. Gonzalo, B.; Vilas, J.L.; Breczewski, T.; Pérez-Jubindo, M.A.; de la Fuente, M.R.; Rodriguez, M.; León, L.M. Synthesis, characterization, and thermal properties of piezoelectric polyimides. J. Polym. Sci. A Polym. Chem. 2009, 47, 722-730.

44. Gutiérrez, J.; Barandiarán, J.M.; Lasheras, A.; Vilas, J.L.; San Sebastián, M.; León, L.M. Improving the performance of high temperature piezopolymers for magnetoelectric applications. Key Eng. Mater. 2013, 543, 439-442.

45. Ryu, J.; Vázquez Carazo, A.; Uchino K.; Kim, H. Magnetoelectric Properties in Piezoelectric and Magnetostrictive Laminates Composites. Jpn. J. Appl. Phys. 2001, 40, 4948-4951.

46. Finkel, P.; Gao, J.; Dolabdjian, C.; Li, J.; Viehland, D. Stress reconfigurable tunable magnetoelectric resonators as magnetic sensors. Appl. Phys. Lett. 2013, 102, doi:10.1063/1.4789500.

47. Gao, J.; Gray, D.; Shen, Y.; Li, J.; Viehland, D. Enhanced DC magnetic field sensitivity by improved flux concentration in magnetoelectric laminates. Appl. Phys. Lett. 2011, 99, doi:10.1063/1.3650713.

48. Dong, S.X.; Bai, F.; Zhai, J.Y.; Li, J.F.; Lu, G.Q.; Viehland, D.; Zhang, S.J.; Shrout, T.R. Circumferential-mode, quasi-ring-type, magnetoelectric laminate composite-a highly sensitive electric current and/or vortex magnetic field sensor. Appl. Phys. Lett. 2005, 86, doi:10.1063/1.1923184.

49. Dong, S.; Zhai, J.; Priya, S.; Li, J.; Viehland, D. Tunable features of magnetoelectric transformers. IEEE Trans. Ultrason. Ferroelectr. Freq. Control 2009, 56, 1124-1127.

50. Shastry, S.; Srinivasan, G.; Bichurin, M.I.; Petrov, V.M.; Tatarenko, A.S. Microwave magnetoelectric effects in single crystal bilayers of yttrium iron garnet and lead magnesium niobate-lead titanate. Phys. Rev. B 2004, 70, doi:10.1103/PhysRevB.70.064416.

51. Fetisov, Y.K.; Srinivasan, G. Electric field tuning characteristics of a ferrite-piezoelectric microwave resonator. Appl. Phys. Lett. 2006, 88, doi:10.1063/1.2191950.

52. Huang, Z. Theoretical modeling on the magnetization by electric field through product property. J. Appl. Phys. 2006, 100, doi:10.1063/1.2388125.

53. Fang, Z.; Lu, S.G.; Li, F.; Datta, S.; Zhang, Q.M.; el Tahchi, M. Enhancing the magnetoelectric response of Metglas/polyvinylidene fluoride laminates by exploiting the flux concentration effect. Appl. Phys. Lett. 2009, 95, doi:10.1063/1.3231614.

54. Jin, J.; Lu, S.G.; Chanthad, C.; Zhang, Q.; Haque, M.A.; Wang, Q. Multiferroic Polymer Composites with Greatly Enhanced Magnetoelectric Effect under a Low Magnetic Bias. Adv. Mater. 2011, 23, 3853-3858.

55. Gutiérrez, J.; Lasheras, A.; Barandiarán, J.M.; Vilas, J.L.; San Sebastián, M.; León, L.M. Temperature response of Magnetostrictive/Piezoelectric Polymer magnetoelectric Laminates. Key Eng. Mater. 2012, 495, 351-354. 
56. Gutiérrez, J.; Lasheras, A.; Barandiarán, J.M.; Vilas, J.L.; San Sebastián, M.; León, L.M. Improving the magnetoelectric response of laminates containing high temperature piezopolymers. IEEE Trans. Magn. 2013, 49, 42-45.

57. Zhai, J.Y.; Dong, S.X.; Xing, Z.; Gao, J.; Li, J.F.; Viehland, D. Tunable magnetoelectric resonance devices. J. Phys. D Appl. Phys. 2009, 42, doi:10.1088/0022-3727/42/12/122001.

(C) 2014 by the authors; licensee MDPI, Basel, Switzerland. This article is an open access article distributed under the terms and conditions of the Creative Commons Attribution license (http://creativecommons.org/licenses/by/3.0/). 